\title{
Mackie-2015 program
}

\section{Thursday July 2, 2015}

08:30 Opening by Prof. dr. ir. Guido Van Huylenbroeck, Dean of the Faculty of Bioscience Engineering, Ghent University.

08:40 Plenary lecture: On the detailed modelling of high temperature nanoparticles synthesis, by Markus Kraft (UK). Session chair: Kevin Van Geem.

09:40 Coffee break.

In room 3 (Blanquaert), parallel session overlapping with ICCK in rooms 1 and 2, session chair Geraldine Heynderickx:

10:00 A heterogeneous multi-scale dynamic model for simulation of catalytic reforming reactors, by Grigorios Pantoleontos (Greece).

10:25 Efficient method for the calculation of rate coefficients of elementary reactions in the gas phase, by Andrey Koksharov (Germany).

10:50 New observation of water gas shift equilibrium in flames, by Wendong Wu (USA).

11:15 On reactive settling of activated sludge, by Raimund Bürger (Chile).

11:40 Parameter fitting: Which algorithm to choose? by Benoît Celse (France).

12:05 Probing pore blocking effects on multiphase reactions at the particle level using a discrete model, by Guanghua Ye (UK / China).

12:30 A comparative study of optimization algorithms for a cellular automata model, by Saurajyoti Kar (India).

12:55 Lunch and Poster session: The Sectional Quadrature Method of Moments (SQMOM): An Application to Liquid-liquid Extraction Columns by Semer Alzyod (Germany); Slow Manifolds identification for dimensionality reduction of chemical kinetics: a computational route by Alessandro Ceccato (Italy); Traits of regularity in stochastic chemical kinetics: analogy with the "Slow Manifolds" feature in deterministic kinetics by Paolo Nicolini (Italy / Czech Republic); Mathematical description of the kinetics of photochemical reactions by Katalin Ôsz; Exact analytical solution of a non-linear reaction-diffusion problem for full range of parameters values - multiplicity and dead zone coexistence and Modeling of gas flow - usefulness of the Laplace transform and CAS-type programs by Mirosłav K. Szukiewicz (Poland).

Session chair for the afternoon: Denis Constales. Location: room Vermeylen.

14:00 Plenary lecture: Quadrature-Based Moment Methods in Chemical Engineering, by Rodney O. Fox (USA).

15:00 Coping with heterogeneity and stochasticity in microbial processes, by Denis Pischel (Germany).

15:25 The switching point between kinetic and thermodynamic control of competitive reactions, by Daniel Branco Pinto (Belgium)

15:50 Fischer-Tropsch Synthesis SSITKA simulation: balancing between model complexity, computational effort and relevance of the included features, by Jonas Van Belleghem (Belgium)

16:15 Novel heuristics for mediating radical chain reactions: a stochastic simulation of the synthesis of copolymers with tailored monomer sequences, by Paul H.M. Van Steenberge (Belgium)

16:40 Calibration And Analysis Of A Direct Contact Membrane Distillation Model Using Monte Carlo Filtering and good modelling practice, by I. Hitsov (Belgium)

Find us on www.mackie-workshops.com and www.facebook.com/mackieworkshops

Mackie-2015 is supported by the FWO Research Foundation Flanders, and Ghent University's Faculty of Engineering and Architecture and Faculty of Bioscience Engineering.

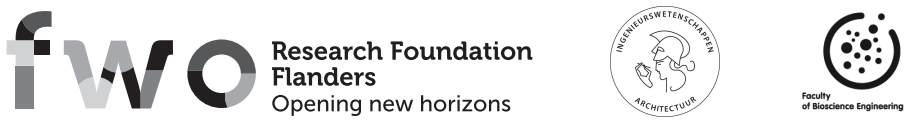




\section{Friday July 3, 2015}

08:40 Plenary seminar: 135 years in chemical kinetics: Bykov-Gorban-Yablonsky's problem, by Valeriy I. Bykov, Alexander N. Gorban and Gregory S. Yablonsky. Session chair: Guy B. Marin. Location: room Vermeylen.

09:40 Coffee break.

Room 1 (Vermeylen), chair: Geraldine Heyn- Room 2 (Blancquaert), chair: Ingmar Nopens derickx

10:00 Optimal design of chemical processes with joint chance constraints, by Gennadi M. Ostrovsky (Russia).

10:25 Simulations of instationary operated heterogeneous catalytic reactions, by Frerich J. Keil (Germany).

10:50 Integration of DFT calculations into microkinetic analysis: Application to carbon dioxide hydrogenation on ceria, by Cynthia S. Lo (USA).

11:15 Explicit formulas for reaction probability in reaction-diffusion experiments, by Renato Feres (USA).

11:40 Stochastic effects in autocatalysis, by Gabor Lente (Hungary).

12:05 Oscillating regimes of first order phase transition by Valeriy I. Bykov (Russia).

Low-dimensional Modeling of Reactions and Transport in Stratified Microflows, by Jason R. Picardo (India).

Modeling the chaotic dynamics of heterogeneous catalytic reactions with fast, intermediate, and slow variables, by N.A. Chumakova (Russia)

Modelling of Influence of Oxygen Bulk Diffusion in Nickel on Oscillatory Kinetics of Catalytic Oxidation of Methane, by V. Ustyugov (Russia).

Application of population balance concept in modeling of FCC riser reactions, by Dariusz S. Orlicki (USA).

Use of Hybrid Monte-Carlo Models in Online Control of Product Quality in Emulsion Copolymerization, by Alexandr Zubov (Czech Republic).

Slow Manifolds identification for dimensionality reduction of chemical kinetics, by Diego Frezzato (Italy).

12:30 Lunch and Mackie Award. Session chair for the plenary lectures: Gregory S. Yablonsky. Location: room Vermeylen.

14:00 Plenary lecture: Lyapunov functions and stability of kinetics: from Boltzmann to present days, by Alexander N. Gorban (UK).

15:00 Plenary lecture: Methods and tools for kinetic model identification, by Rafiqul Gani (Denmark). Chair Rafiqul Gani:

16:00 Lack of fit and degrees of freedom in kinetic modelling, by Bengt Andersson (Sweden).

16:25 The nature of vortex breakdown, by V. Shtern (U.S.A.).

Find us on www.mackie-workshops.com and www.facebook.com/mackieworkshops

Mackie-2015 is supported by the FWO Research Foundation Flanders, and Ghent University's Faculty of Engineering and Architecture and Faculty of Bioscience Engineering.

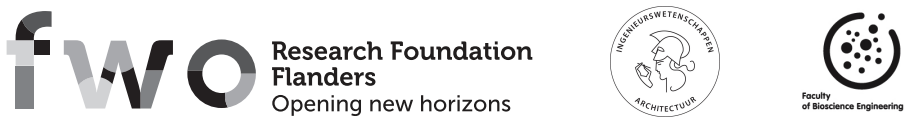

